

\title{
VALORACIÓN DE ACTIVOS, EFICIENCIA DE MERCADOS FINANCIEROS Y CASOS RELACIONADOS CON LA RACIONALIDAD E IRRACIONALIDAD DE LOS AGENTES. UNA REVISIÓN BIBLIOGRÁFICA
}

\author{
VALUATION OF ASSETS, EFFICIENCY OF FINANCIAL MARKETS \\ AND CASES RELATED TO THE RATIONALITY AND IRRATIONALITY \\ OF THE AGENTS. A BIBLIOGRAPHIC REVIEW
}

\begin{abstract}
Autor 1
Joan Sebastián Rojas Rincón

Magíster en Contabilidad y Finanzas, Magíster en Mercadeo, Especialista en Administración Financiera, Especialista en Gerencia Estratégica de Mercadeo, Administrador de Empresas, Tecnólogo en Administración Bancaria y de Instituciones Financieras - Universidad Nacional Abierta y a Distancia

ORCID: https://orcid.org/0000-0002-1203-9979

Correo electrónico: joan.rojas@unad.edu.co
\end{abstract}

\section{Autor 2}

Sara Liliana Martínez Cuervo

Especialista en Administración Financiera, Contadora Pública - Universidad

Nacional Abierta y a Distancia

ORCID: https://orcid.org/0000-0002-7409-7461

Correo electrónico: sara.martinez@unad.edu.co

\section{Autor 3}

Carlos Andrés Avellaneda Sánchez

Administrador de Empresas, Estudiante de Tecnología en Producción Agrícola Universidad Nacional Abierta y a Distancia

ORCID: https://orcid.org/0000-0002-6446-7449

Correo electrónico: caavellanedasa@unadvirtual.edu.co

\section{Autor 4}

Karen Nayibe Serrano Ortiz

Estudiante de Administración de Empresas, Tecnólogo en Contabilidad y

Finanzas - Universidad Nacional Abierta y a Distancia

ORCID: https://orcid.org/0000-0002-2896-0156

Correo electrónico: knserranoo@unadvirtual.edu.co 




\section{RESUMEN}

Este trabajo tiene como objetivo explorar la teoría de los mercados financieros eficientes, bajo un marco de análisis basado en el supuesto de racionalidad de los agentes. Para tal fin, se parte de un análisis de las consideraciones teóricas y de las implicaciones prácticas en la operatoria del análisis bursátil y toma de decisiones de inversión. Luego, a partir de una revisión documental, se abre una discusión alrededor de la pregunta problematizadora: ¿Son eficientes los mercados financieros? El punto de partida es el planteamiento de los niveles de eficiencia propuestos por Fama (1970) para que, a partir de estos, se pueda librar la discusión alrededor de la evidencia empírica de algunos estudios o casos dados en la práctica, con los que se plantea serios cuestionamientos a este enfoque teórico. Al final, se plantean algunas consideraciones desde la óptica de las finanzas conductuales, donde los sentimientos, las emociones y los patrones de comportamiento colectivo pueden explicar ciertas anomalías en los mercados.

Palabras clave: finanzas, eficiencia de los mercados financieros, valoración de activos financieros, análisis fundamental, análisis de mercado, finanzas conductuales.

\section{ABSTRACT}

This work aims to explore the theory of efficient financial markets, under an analysis framework based on the assumption of rationality of the agents. For this purpose, it starts from an analysis of the theoretical considerations and the practical implications in the operation of the stock market analysis and investment decision making. Then, based on a documentary review, a discussion is opened around the problematizing question: Are financial markets efficient? The starting point is the approach to the efficiency levels proposed by Fama (1970) in order, based on these, to free the discussion around the empirical evidence of some studies or cases given in practice, with which serious questions are raised. to this theoretical approach. In the end, some considerations are raised 


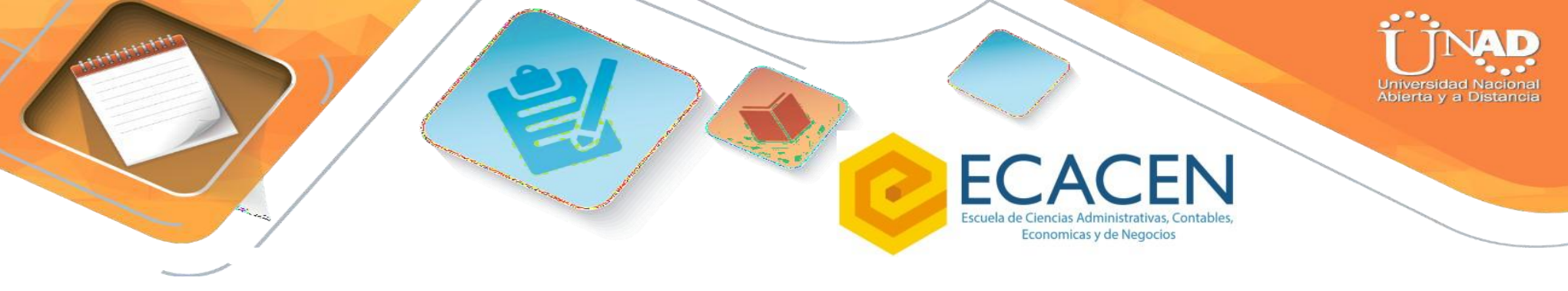

financieros, el uso de los insumos de información y la identificación de algunas anomalías, de donde se desprende la discusión y conclusiones del documento.

\section{METODOLOGÍA}

El presente trabajo de investigación tiene un enfoque exploratorio, en el que, a partir de una revisión documental realizada de forma sistemática, se analizan algunos casos relacionados con el comportamiento de los agentes en los mercados financieros. De acuerdo con Bowen (2009), al llevar a cabo un análisis documental, el investigador debe ser capaz de demostrar la capacidad de identificar la información pertinente y separarla de la que no lo es; por tanto, para los fines asociados al desarrollo de esta investigación, se plantea llevar a cabo este ejercicio sistemático con fundamento en un marco de referencia. Considerando lo anterior, con soporte en el marco teórico y conceptual planteado, se desarrolla una discusión académica en torno al concepto de eficiencia de los mercados y sus implicaciones relacionadas con la toma de decisiones de inversión basadas en el análisis técnico o a partir de los fundamentales. Así las cosas, el presente trabajo se construye a manera de un artículo de revisión, donde se sintetizan los hallazgos de trabajos relacionados con los mercados financieros en diferentes fuentes bibliográficas.

Para la construcción de este artículo se lleva a cabo un proceso sistemático de revisión de literatura; de tal manera que en un primer momento se realiza una indagación en diferentes bases de consulta científica, a partir de palabras clave como mercados financieros, eficiencia de los mercados y valoración de activos financieros. A partir de este ejercicio de indagación, se procede a identificar aquellos documentos, particularmente que tratan el fenómeno de eficiencia de los mercados financieros. Alrededor de una discusión relacionada con los dos principales enfoques de análisis de información, como lo son el análisis técnico y el análisis basado en los fundamentales, se desarrolla una discusión alrededor de la pregunta: ¿Son eficientes los mercados financieros? Finalmente, se analizan algunos casos de estudio que respaldan la 


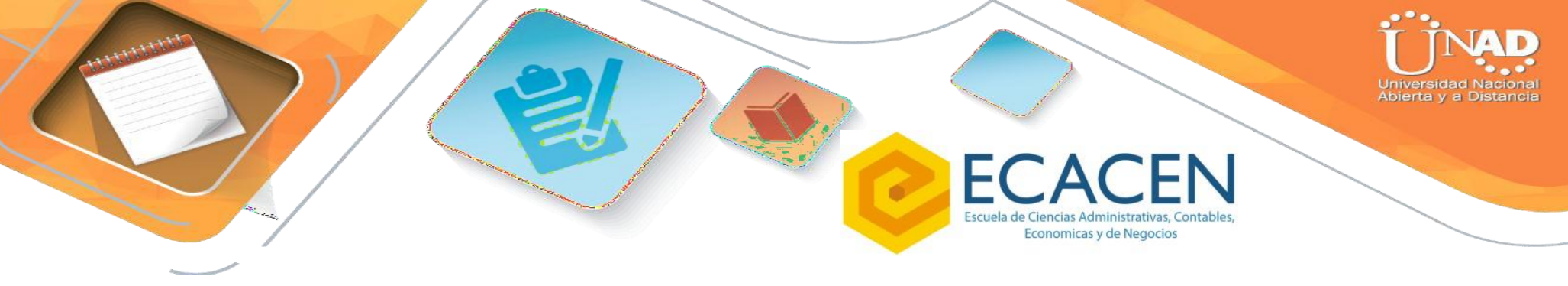

hipótesis de eficiencia de los mercados o, por el contrario, dan cuenta de algunas anomalías en el comportamiento de los agentes que permiten desarrollar la discusión desde la perspectiva de las finanzas conductuales. Con el fin de desarrollar estas fases, se acoge la propuesta de Miles y Huberman (1994) para el desarrollo de trabajos de revisión bibliográfica, donde se parte de la recolección de los datos, la reducción de los mismos, la presentación y análisis de información y la búsqueda de conclusiones, con enfoque interactivo orientado a la construcción dinámica de conocimiento.

\section{DISCUSIÓN Y RESULTADOS}

\section{Enfoques para el Análisis de Información Financiera}

En el ámbito de las inversiones en activos financieros, se han consolidado dos enfoques para operar en los mercados. Por un lado, las decisiones pueden respaldarse con base en información que proveen los fundamentales; es decir, soportadas en las características particulares de un activo y la información financiera que permite evaluar su desempeño. Por otro lado, para la toma de decisiones de inversión puede recurrirse al análisis de información relacionada con los precios de dichos activos, especialmente, la que tiene que ver con tendencias y patrones que representan los movimientos del mercado. De acuerdo con Sharpe (2003), el proceso de inversión implica aspectos como la manera en que un inversionista toma decisiones sobre en qué invertir en el mercado de valores negociables, que tan vastas deben ser estas inversiones y cuando hacerlas. Lo anterior, significa que el análisis financiero orientado a la inversión permite identificar los activos de los que se espera obtener los mejores rendimientos o que contribuyen a diversificar el riesgo en una cartera de inversión. También debe evaluarse el volumen de las transacciones y el momento adecuado para tomar una posición en el mercado. 


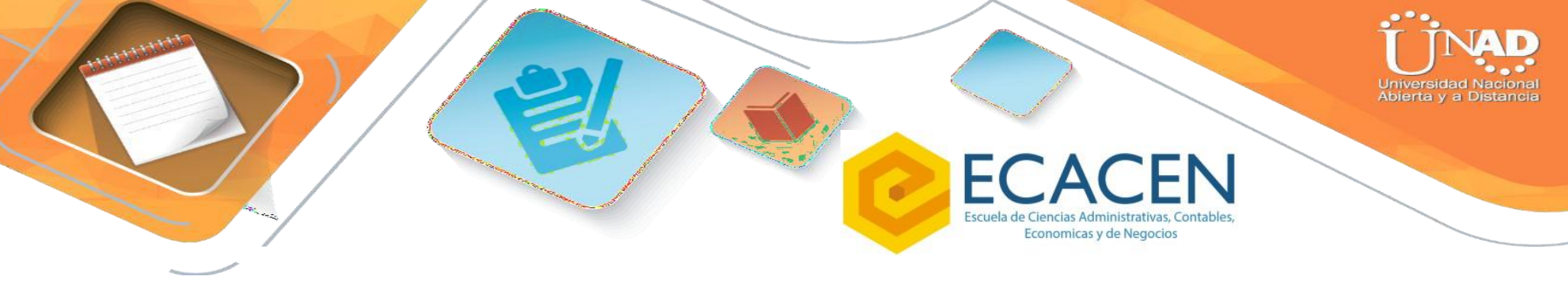

En líneas generales, la toma de decisiones de inversión implica asumir riesgos considerables; por lo que la teoría financiera supone que los agentes tienen aversión al riesgo y que, por ende, racionalizarán el proceso de toma de decisiones, con la finalidad de gestionar el riesgo al que se exponen. Sin embargo, estudios recientes han demostrado que el comportamiento del inversionista no necesariamente es racional. Lo anterior, conduce a que se generen anomalías en los mercados financieros y prueba de ello son las burbujas especulativas que pueden definirse como una

situación en la que la noticia del aumento en los precios estimula el entusiasmo de los inversores, y eso se extiende de una persona a otra a modo de contagio $y$, en el proceso, amplifica noticias que podrían justificar el aumento de precios y atrae a grupos cada vez más numerosos de inversores que, a pesar de sus dudas sobre el valor real de la inversión, se ven atraídos hacia la misma. (Shiller, 2013).

Así bien, a pesar de que se espera que el inversionista procure acceder a la información suficiente y relevante para la toma de decisiones, por cuanto está asumiendo riesgos respecto a los capitales implicados en una operación de inversión, los mercados financieros se caracterizan por tener considerables imperfecciones. Lo anterior no solo se da en razón de las asimetrías de información; sino también, por la misma naturaleza del ser humano, en el sentido en que las decisiones también son inducidas por las emociones, por la intuición o por patrones de comportamiento colectivo. Por contraste, cuando las decisiones son tomadas con base en información de mercado o del activo en particular, generalmente se adoptan dos enfoques de análisis.

\section{En función de los fundamentales}

Los fundamentales, en el ámbito de las finanzas, permiten analizar el estado actual de una situación inversión $y$, con un buen análisis e interpretación, posiblemente también contribuya al análisis de estados 


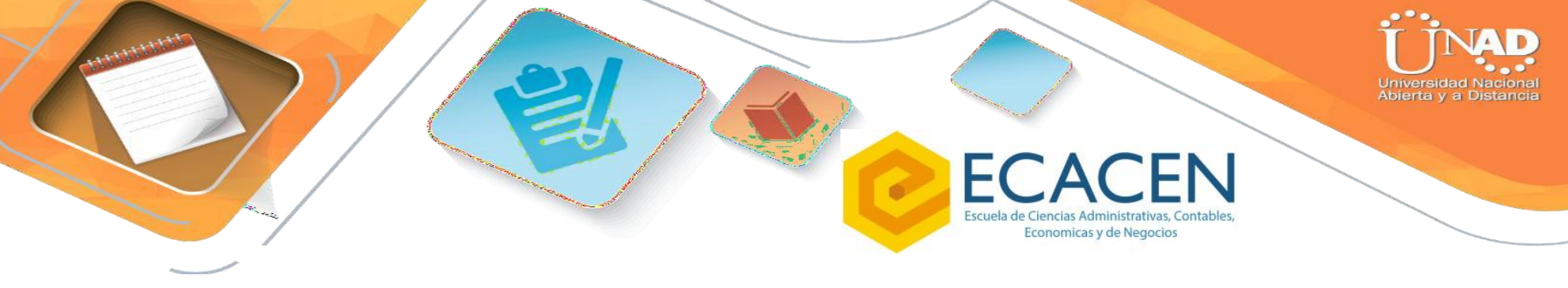

futuros. Desde el punto de vista financiero, los fundamentales serían variables que logran explicar la situación y el desempeño de un activo financiero. Por tanto, cuando se habla del análisis de los fundamentales, se está haciendo referencia al proceso que permite indagar acerca de los móviles del precio de un activo, por ejemplo, el precio de una acción. En este caso, Graham \& Dodd (2009), plantean que, analizar estos valores, implicar analizar un negocio; por lo que se trata realmente de emprender un estudio que debe ser llevado con cuidado con un nivel importante de detalle. Eso significa que deben estudiarse aquellas variables que explican la situación actual de la empresa que colocó dichas acciones en el mercado y que pueden explicar los cambios de esa situación en el corto, mediano y largo plazo. De esta forma, es posible valorar la empresa teniendo en cuenta sus activos actuales y el pronóstico de sus rendimientos financieros futuros. Se entiende, entonces, que una acción, al ser parte de la empresa, tiene un valor actual proporcional al valor total de la empresa.

El valor de una acción basado en el análisis de los fundamentales es el valor intrínseco de la acción, también conocido como el valor teórico, aunque bien, cada agente en el mercado puede tener uno diferente según la forma particular en que lo estime, o las diferentes variables cuantitativas y cualitativas que incluya en el análisis. Sin embargo, el método común es el cálculo del valor presente de los rendimientos futuros de la acción, o sea, el valor presente de los dividendos por pagar de la empresa, pero es un modelo condicionado, ya que las expectativas de los pagos futuros se forman únicamente con la información disponible en un determinado momento en el tiempo (Nieto \& Rodríguez, 2005). En ese sentido, el valor intrínseco se definiría como una promesa de flujos futuros (Vilariño, 2013), y el pago de dicho valor daría derechos sobre los ingresos por venir de la compañía.

Las variables a tener en cuenta pueden ser divididas en variables macroeconómicas, de la industria y de la compañía (Radim, 2012). La relación entre variables internas y externas es una característica esencial del proceso; dado que el valor de una compañía y, por ende, de una 


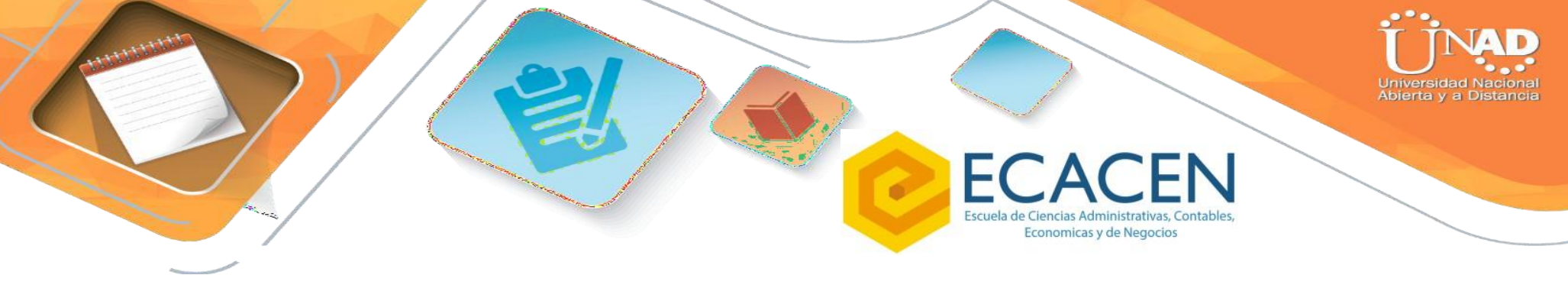

acción, no depende únicamente de la gestión individual de esta, sino también de factores externos que deben entrar en el análisis, que son relativos a la industria específica en la que se encuentra y los temas macroeconómicos concernientes al lugar o lugares donde opera. En tal sentido, los fundamentales son un conjunto de muchas variables $\mathrm{y}, \mathrm{a}$ pesar de que hay algunas más relevantes que otras, cada analista puede evaluar, según su criterio y sus necesidades, cuáles tomar en cuenta y qué peso asignarles en el análisis; así como la forma particular de analizarlas. Al respecto, Vilariño (2013) menciona que los modelos para valorar un activo basados en los fundamentales son discrecionales, donde lo que determina los flujos de caja futuros es una opinión sobre la información disponible, guiada por intereses particulares. Considerando lo anterior, si bien con el análisis de inversiones basado en los fundamentales se pretende ser objetivo; no puede desconocerse la perspectiva del analista y, de ahí, las diferentes estimaciones del valor financiero a las que puede llegarse.

\section{En función de los precios o cotizaciones}

Este enfoque es conocido, generalmente, como análisis técnico. Se trata de un análisis del comportamiento del precio de un activo en el mercado, caracterizándose por ser empírico y con fundamento en herramientas gráficas, estadísticas y matemáticas. Para tal fin, puede hacerse uso de modelos econométricos o indicadores técnicos que permiten hacer un seguimiento de las tendencias en el mercado, como lo es el caso de las gráficas de velas japonesas, los promedios móviles, el suavizamiento exponencial o herramientas más sofisticadas para medir el impulso como el indicador MACD (Media Móvil de Convergencia/Divergencia). Estas herramientas o indicadores dan cuenta de la evolución de los precios de un activo, el volumen transaccional, entre otros aspectos relevantes para el análisis de los movimientos del mercado. Estos métodos heurísticos o estadísticos pueden ser útiles para identificar periodos de sobrecompra y sobreventa de los activos; que pueden ser respaldados en información proveniente de los fundamentales o en un comportamiento irracional de 


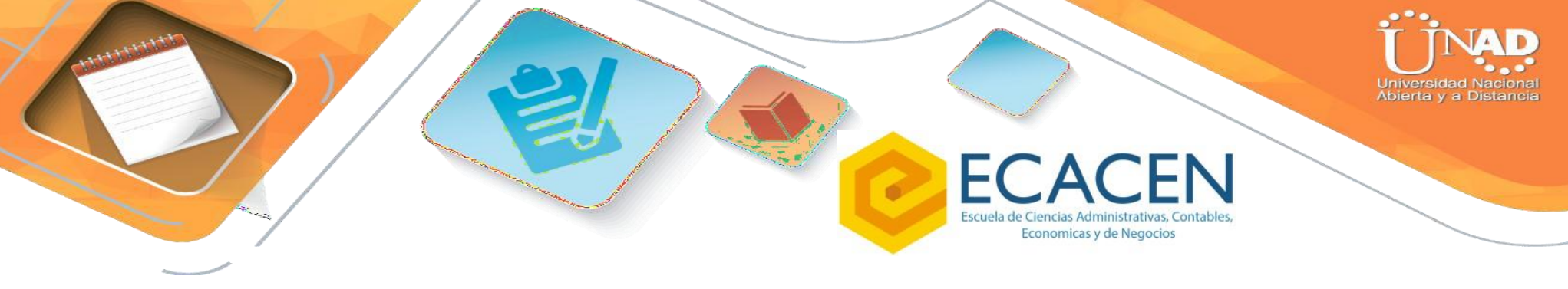

los agentes. En cualquier caso, el operador técnico no se interesa por los móviles que afectan los precios, sino por la tendencia en sí.

Generalmente, las gráficas son la herramienta por excelencia de la que hace uso el analista para identificar tendencias y patrones del precio de la acción en el tiempo, con el objetivo de predecir futuras tendencias en el precio, visibilizar las ganancias en ciertos mercados y demás (Zuluaga, \& Velázquez, 2007). Sobre estas gráficas se aplican algunas herramientas que permiten llevar a cabo análisis con un enfoque más heurístico, es decir, con fundamento a partir reglas, comúnmente conocidas como reglas de dedo; las cuales, generalmente, se justifican con base en la experiencia. Por ejemplo, a partir de ciertos patrones, se grafican líneas de soporte o resistencia, líneas de tendencia, canales por donde transita el patrón de comportamiento. Así mismo, se estudian patrones comunes, que generalmente se clasifican en patrones de cambio o de continuidad. En resumen, el análisis técnico es aquel que se enfoca en el seguimiento al comportamiento de los precios $y$, en líneas generales, estudia los movimientos del mercado, "principalmente mediante el uso de gráficos, con el propósito de pronosticas las futuras tendencias de los precios" (Murphy, 2000). Al final, las conclusiones derivadas de un análisis técnico revelan si la tendencia es alcista o bajista y si es el momento para tomar una posición, salir de una posición o mantenerse.

Aunque este enfoque de análisis de inversión se concentra en el estudio la cotización de la acción, el volumen, el interés abierto, las tendencias correspondientes y la premisa de que la historia se repite; también puede verse influenciado en gran medida por la propia perspectiva, racionalidad y factor psicológico del agente que opera y como se anticipa a la actuación de los demás. De acuerdo con Murphy (2000), no puede desconocerse la realidad de que la interpretación de los gráficos es muy subjetiva; por lo que siempre habrá un elemento de duda y desacuerdo. Esto significa que las decisiones de los operadores técnicos están influenciadas por factores que van más allá de las señales que generan los precios; teniendo en cuenta, también, que 


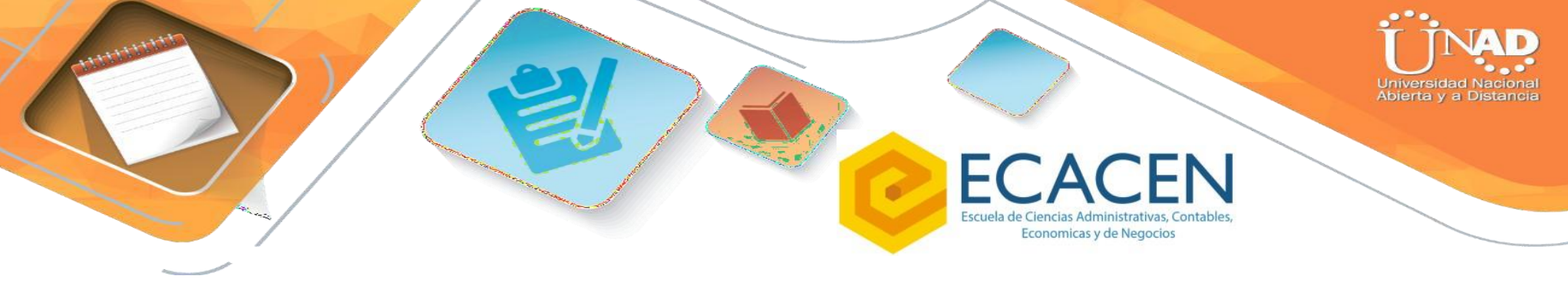

sin embargo, la falta de información en algunos mercados no significa que no son eficientes, simplemente que su eficiencia no es tan fuerte. En Fama (1991), el autor plantea dos formas de aproximarse a la eficiencia de los mercados; por un lado, la versión extrema en la que los precios reflejan toda la información disponible y el costo de acceder a esta información y los costos de transacción son cero. Sin embargo, también se plantea una hipótesis más flexible, "que dice que los precios reflejan información hasta el punto en que los beneficios marginales de actuar sobre la información (las ganancias que se obtienen) no exceden los costos marginales" (Jensen, 1978 citado por Fama, 1991). Así las cosas, con fundamento en la hipótesis de los mercados financieros eficientes, únicamente con el acceso a información privilegiada es posible obtener rendimientos excepcionales al operar en los mercados financieros; por lo que, "si un inversionista no tiene información especial, no importará lo inteligente que sea, no podrá mejorar el rendimiento de su portafolio de inversión" (Daniel \& Titman, 1999).

Desde la perspectiva de la eficiencia de los mercados, el insumo esencial para obtener rendimientos excepcionales en el mercado es la información; sin embargo, del enfoque también se deduce que estas oportunidades son bastantes limitadas y en un nivel de eficiencia extrema, deberían ser prácticamente inexistentes. En el caso de la eficiencia débil, si bien, puede tenerse acceso a las series históricas de precios y rendimientos de los activos, dicho conocimiento expuesto a través del análisis no resulta útil para obtener rendimientos superiores al mercado, ya que el precio sigue un camino aleatorio, $y$, por tanto, es imposible su predicción. Ahora bien, desde el punto de vista del análisis fundamental, podrían encontrarse oportunidades en los mercados financieros si los precios únicamente reflejan la información histórica y no capturan la totalidad de información pública que circula en el mercado. Estas oportunidades para obtener rendimientos anormales estarán dadas, por lo menos hasta que el precio converja en una especie de equilibrio que incorpore dicha información pública (valor intrínseco consensuado). En razón de lo anterior, con un nivel de eficiencia débil se pone en tela de juicio la utilidad del análisis técnico; más aún cuando "se han probado muchas otras reglas de 




transacciones a partir de información privilegiada; pues se considera como una especie de trampa o desequilibrio en las condiciones que tienen los agentes a la hora de operar en los mercados. Algunos estudios han demostrado que la especulación en los mercados financieros, con base en esta información, es más común de lo que parece; lo que conduce a invalidar la hipótesis fuerte de los mercados financieros eficientes. Es posible, también, que uno de los incentivos de quienes siguen a líderes de opinión o influenciadores en los mercados es el posible acceso que estos tienen a información privilegiada; con base en la cual se espera obtener un desempeño superior.

\section{Una eficiencia cuestionada}

Paradójicamente, a mayor disponibilidad de información en los mercados, estos se hacen más eficientes, pero las oportunidades de obtener beneficios se reducen. El cuestionamiento que surge, entonces, es sobre cuál es el incentivo que persigue un inversionista al operar en mercados financieros que se presumen eficientes. Esto, por cuanto, como propone Quiroga (2017), si un mercado tiene un nivel de eficiente débil, entonces no vale la pena hacer análisis técnico o chartista, pues los precios incluyen toda la información histórica de la que se derivan las tendencias. En el mismo sentido bajo una hipótesis semi-fuerte no tendría sentido hacer análisis fundamental y si la eficiencia es fuerte, ni siquiera sería necesario buscar nueva información. Sin embargo, lo que aumenta la eficiencia de los mercados es justamente la constante búsqueda de información y los análisis de técnico y fundamental, que llevan a cabo los analistas financieros; ya que es la competencia por nueva información y por rendimientos a través del arbitraje, lo que logra llevar los precios de los activos hacia su valor real en el mediano y largo plazo. Adicionalmente, ya que los avances tecnológicos han abaratado los costos de transacción y han hecho que la información se conozca más rápido, se espera que dicha información se incorpore antes a los precios de los activos $y$, al menos en el mediano y largo plazo, se converja hacia el precio teórico o de equilibrio. 


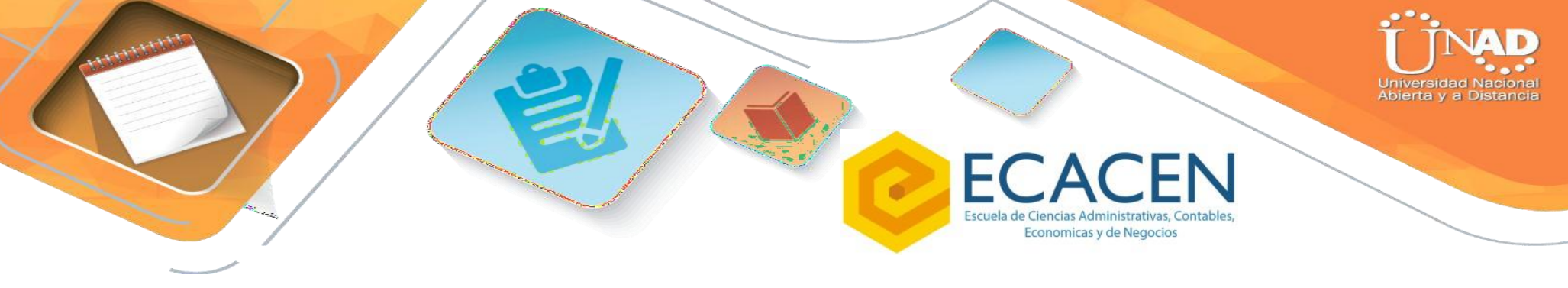

Si bien, los desarrollos tecnológicos pueden inducir a un mayor acceso a información en menor tiempo, también debe pensarse en la otra cara de la moneda. Al respecto, Brad et al. (2001) señalan que aunque el internet está cambiando la forma y los medios en que la información es entregada a los inversores y estos actúan frente a dicha información, logrando reducir los costos fijos y marginales de los servicios financieros; también está contribuyendo a generar condiciones propicias para mantener por largo tiempo errores en los precios de los activos y burbujas especulativas, ya que la relación de inversores sin experiencia frente a inversores con experiencia ha aumentado. Este aumento puede deberse a que el acceso a los mercados financieros es cada vez mayor, y lo será aún más en el futuro, pues va de la mano con el crecimiento de la denominada "tecnología financiera" (Fintech). Estos errores persistentes en los precios y las burbujas especulativas son anomalías del mercado, y algunas suelen presentarse con relativa frecuencia, lo que implica que los mercados no son eficientes, o lo son únicamente durante ciertos lapsos. En un estudio adelantado por Uribe y Ulloa (2011), en el que se estudia la eficiencia en diez mercados desde 2001 y hasta 2010, obtienen como conclusión que, de los diez mercados analizados, solo dos eran eficientes en su versión débil, cuatro eran parcialmente eficientes en su versión débil, y cuatro eran mayormente ineficientes, encontrándose entre estos últimos, los mercados de Estados Unidos e Inglaterra.

La hipótesis de los mercados eficientes parece diluirse en la práctica, y esto puede estar ligado a los conceptos de agente representativo y expectativas racionales a las que refiere Lobejón (2011) como hipótesis irreales; ya que el autor no considera que haya un agente que pueda representar a todos los demás, pues cada persona toma decisiones de forma diferente y sobre diversos fundamentos. Del mismo modo, tampoco ve posible que siempre se tomen decisiones de forma racional o "correcta", por lo que las expectativas de los agentes tampoco serán siempre racionales. Ante esto, puede decirse que, aunque los teóricos tradicionales de las finanzas parecen respaldar la idea de que los mercados financieros son eficientes, al menos hasta cierto punto, nuevas posturas teóricas hacen cuestionable este hecho; ya que, por ejemplo, "la 


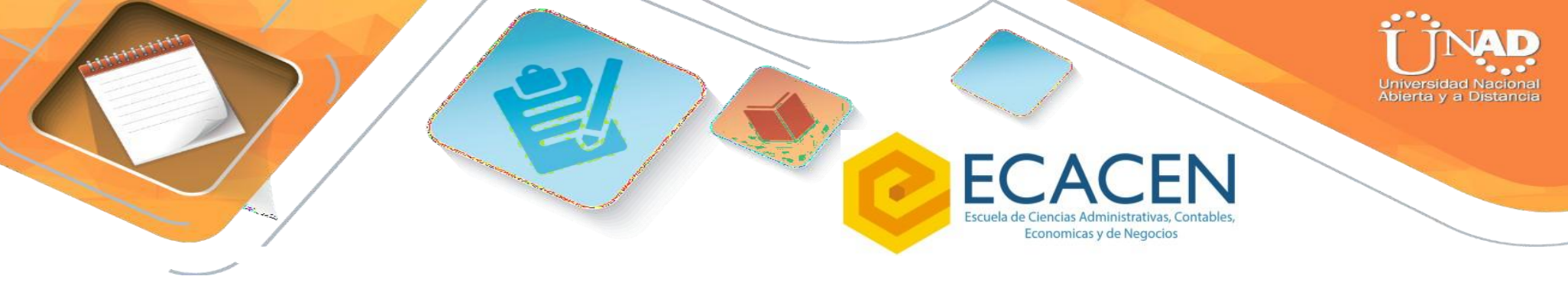

literatura de la psicología describe una miríada de sesgos de comportamiento que potencialmente pueden explicar casi cualquier desviación observada de la hipótesis del mercado eficiente" (Daniel y Titman, 1999).

\section{Un nuevo enfoque para entender los mercados. Las finanzas conductuales}

El comportamiento de los agentes en los mercados financieros ha despertado interés en diversos ámbitos de discusión académica, que van más allá de los tradicionales modelos axiomáticos en los que se fundamenta la teoría económica neoclásica. Es así que autores como Thaler (1980) señala que en ciertas situaciones los consumidores actúan de una manera que es incompatible con la teoría económica, por lo que si se hace un análisis con fundamento en esta se cometerán errores sistemáticos para predecir el comportamiento. En contraste con el supuesto de agente perfectamente racional, aparece el término racionalidad limitada, "el cual se utiliza para designar una elección racional que tiene en cuenta las limitaciones cognitivas del tomador de decisiones: limitaciones tanto del conocimiento como de la capacidad computacional" (Simon, 1990). Por lo anterior, deben tenerse en cuenta las limitaciones de la teoría económica tradicional para estudiar ciertas anomalías que ocurren en los mercados financieros; las cuales, presuntamente están motivadas por comportamientos irracionales, es decir, por limitaciones o sesgos cognitivos del tomador de decisiones. Con fundamento en este hecho, se desarrolla un nuevo campo de conocimiento conocido como economía conductual y de ahí, las finanzas del comportamiento. De esta manera, se propone un nuevo enfoque para el análisis de las finanzas, desde una perspectiva psicológica, centrada, fundamentalmente, en el análisis del comportamiento de las personas.

Las finanzas conductuales surgen como un enfoque que centra su atención cómo se comportan realmente los inversionistas; en contraste con las finanzas tradicionales, que se soportan en la teoría de como debería ser este comportamiento. De acuerdo con lo que señala Hosseini 




embargo, el planteamiento del autor está soportado en el hecho de que, si los mercados financieros no son eficientes, entonces habría inversionistas que se comportan de manera irracional y, por tanto, aquellos que si aprovechan la información deberían obtener rendimientos superiores. Sin embargo, no queda claro, entonces, como un mercado eficiente da cabida a la formación de burbujas especulativas que pueden sostenerse sobre un horizonte significativo de tiempo. ¿Es que acaso hay momentos en que los agentes, en general, tienen un comportamiento irracional?

\section{Racionalidad Imperfecta}

Por contra al planteamiento de eficiencia de los mercados financieros; un vasto cuerpo de literatura, respaldado por evidencia empírica, da cuenta de diversas anomalías que ocurren en el campo de las inversiones. Algunos autores plantean que la formación de burbujas puede explicarse a raíz de la falta de calidad en la información de la que dispone el analista, como es el caso de los reportes contables; "donde se reconoce imperfecciones inevitables por dificultades de medición" (Penman, 2003). Por otro lado, son muchos los ejemplos sobre actuaciones irracionales de los agentes y que contrastan con la hipótesis de eficiencia de los mercados, como. Dentro de los ejemplos más recientes, puede citarse la burbuja hipotecaria de 2008, que da cuenta de una clara anomalía del mercado, y se da por falta de rigurosidad en los análisis de mercado y de fundamentales, así como por un comportamiento irracional de los inversores, lo que va contra la hipótesis de eficiencia. De acuerdo con Shiller (2008), Alan Greenspan, en un artículo de opinión publicado en Financial Times, reconoció que en la formación de la burbuja especulativa había habido euforia; pero el problema esencial, radica en que los modelos de análisis de riesgos y los modelos econométricos; por más complejos que se hubieran vuelto, aún resultaban demasiado simples para capturar las diferentes variables que impulsan la realidad económica global. Lo anterior, justifica una vez más el hecho de que el mundo social que es objeto de análisis por parte de los economistas es demasiado complejo y resulta imposible reducirlo a un modelo. 


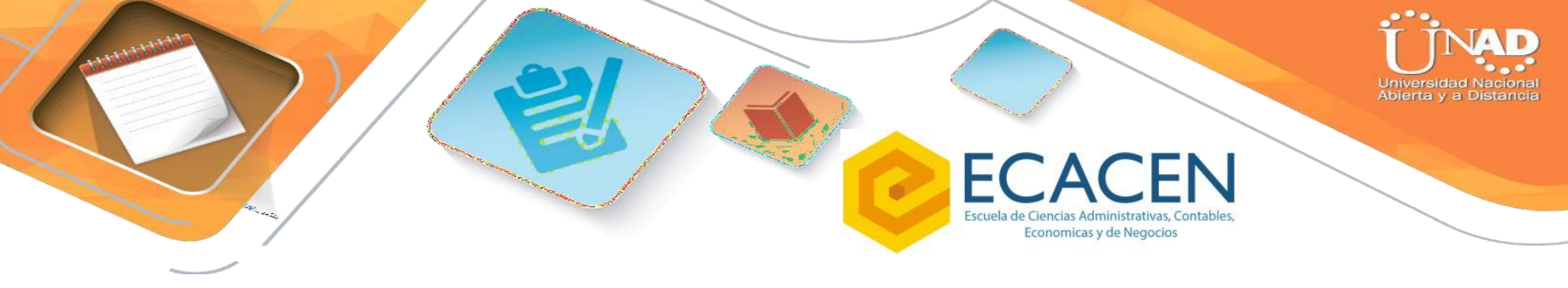

Por su parte, los mercados de activos tecnológicos son reconocidos por ser altamente volátiles; lo que conduce a que sean buscados recurrentemente por inversionistas con un mayor nivel de tolerancia al riesgo, quienes están dispuestos a realizar inversiones especulativas. Sin embargo, entrando a la primera década del nuevo milenio, se acrecienta en el mercado un interés sin precedentes por las firmas de base tecnológica, especialmente, aquellas que configuran su modelo de negocios sobre la base de las prestaciones que ofrece el internet. Estas son conocidas como las empresas dot.com o las punto.com y la exuberancia alrededor de los potenciales beneficios que se esperaban de esta nueva forma de hacer negocios y el consecuente desplome de los precios es un hecho bastante documentado en la literatura financiera. Algunas condiciones prácticas en los mercados financieros podrían explicar este hecho; puesto que debido a que la toma de posiciones cortas es limitada, "las creencias de los inversores pesimistas se vieron abrumadas por las creencias optimistas, lo que llevó a la alta valoración de las acciones de internet" (Ofek \& Richardson, 2003). Una vez más, las condiciones contextuales y psicológicas inciden en las percepciones de los agentes, respecto al desempeño de los activos financieros.

Las limitaciones en el acceso y la calidad de la información son condiciones que inducen a que los inversionistas tomen decisiones en base a expectativas irreales. Si bien, esto no significa que los inversionistas no elaboren sus procesos de decisión a partir de procedimientos lógicos y sistemáticos, es decir, con fundamento en la racionalidad; al final, la información no es completamente accesible, por lo que se sigue afrontando el hecho de que se tiene racionalidad limitada. En otras palabras, la racionalidad no solo está limitada por sesgos cognitivos, sino que también influye la posibilidad de acceso a la información y la disponibilidad de tiempo para tomar una decisión. Al respecto, pueden mencionarse algunos casos como el de Enron Corporation. Este caso ha sido considerado como uno de los mayores escándalos financieros de la historia; por cuanto se cometió una defraudación contable, en el sentido en que la información había sido maquillada, reflejando una situación 


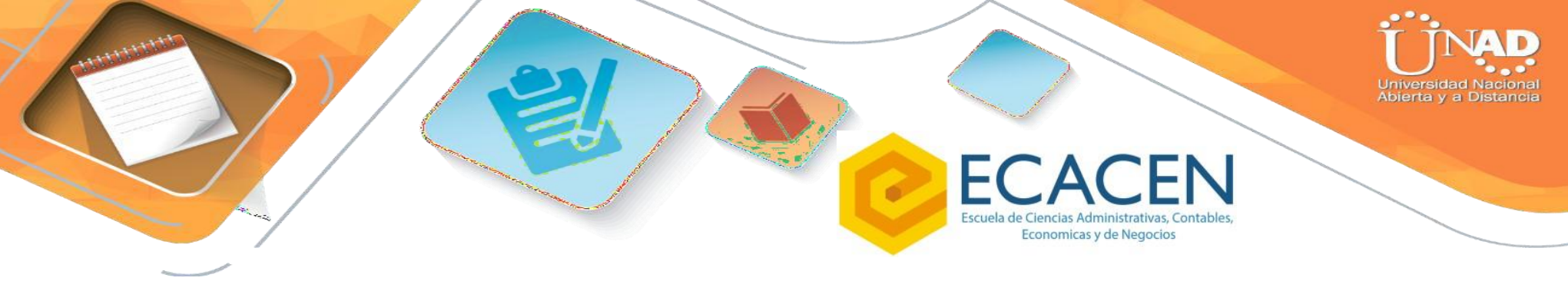

de los estados financieros" (Cardona et al., 2015). Así como estos casos, otros dan cuenta de importantes anomalías en los mercados, derivadas de las asimetrías de información y la búsqueda de beneficios personales que no siempre tienen un efecto favorable sobre el bien común.

\section{CONCLUSIONES}

En el presente artículo de revisión se plantea como categoría de análisis la Hipótesis de los Mercados Financieros Eficientes, sobre la cual se lleva a cabo una discusión en razón del trasfondo teórico y de las implicaciones prácticas. Se parte del hecho que en el estudio de las finanzas "se supone que los mercados son perfectos o totalmente eficientes, con objeto de construir un modelo de decisión" (Mascareñas, 2007). Lo anterior implica que el cuerpo teórico de las finanzas se fundamenta alrededor de suposiciones del comportamiento de los agentes y de los mercados en sí mismos; asumiéndose ausencia de costos de transacción, racionalidad en la toma de decisiones y mercados completos. Sin embargo, un buen compendio de evidencia empírica plantea una seria discusión sobre el entramado teórico en el que soportada la moderna teoría financiera; pues son observables considerables anomalías que hacen cuestionables los supuestos tradicionales de la hipótesis de eficiencia.

Si bien, la eficiencia de los mercados financieros no es un concepto absoluto, en el sentido en que generalmente se asigna una escala de graduación, pudiendo ser, por tanto, fuerte como también débil; se ha documentado que, incluso, la hipótesis de eficiencia débil también es cuestionable. Teniendo en cuenta este estado del arte en el que se basa la discusión llevada a cabo a lo largo de este documento; no puede desconocerse, la naturaleza del ser humano y sus implicaciones en la toma de decisiones. Por tanto, si bien existe una fundamentación de base sobre el cómo deberían comportarse los agentes en el mercado y cuál sería, entonces, el precio que se espera se forme a partir de tal comportamiento que se asume como racional; también es importante que el inversionista sea capaz de discernir sobre aquellos momentos en que 


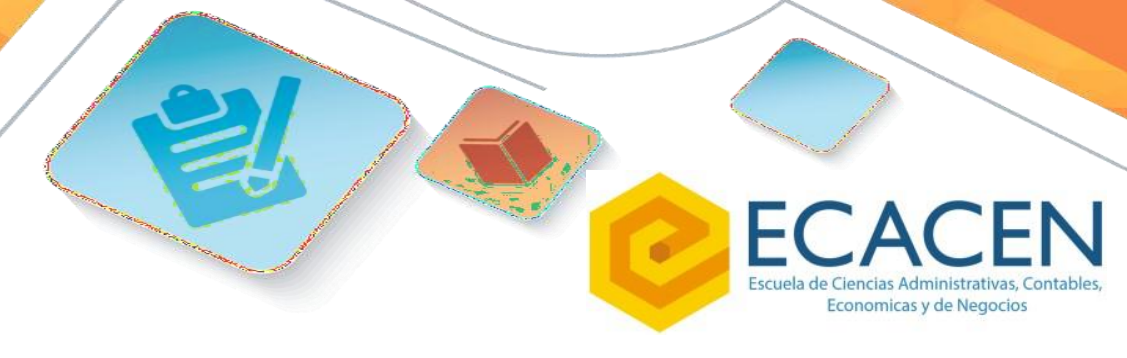

la euforia colectiva o el miedo están influenciado la dinámica de los mercados. Al respecto, Gitman \& Joehnk (2009) proponen que algunos activos son fundamentalmente fuertes y otros débiles, por lo que un inversionista debería poder distinguir entre estos tipos. En líneas generales, un inversionista debe ser consciente de que los mercados no siempre operan bajo condiciones de eficiencia $y$, por tanto, debería evaluar el contexto temporal y espacial a fin de identificar momentos en los que el mercado está operando bajo exuberancia irracional.

\section{REFERENCIAS}

BBC Mundo. (2011). La locura de los mercados. La economía es ya cosa de psicólogos. BBC News. https://www.bbc.com/mundo/noticias/2011/08/110811 economia finanzas psicologia mercados az

Brad, M. Barber \& Terrance Odean. (2001). The Internet and the Investor. The Journal of Economic Perspectives, 15(1), 41-54.

Cardona, L., Varón, M. \& Arias, S. (2015). El caso Interbolsa. Analizado desde la problemática contable. Libre Empresa, 24, pp.141-162.

Daniel, K. \& Titman, S. (1999). Market efficiency in an irrational world. Financial Analysts Journal, 55(6), 28-40.

Fama, E. (1970). Efficient capital markets. A review of theory and empirical work. Journal of Finance, 25(2), 383-417

Fama, E. (1991). Effciente capital markets II. Journal of Finance, 46(5), 1575-1617.

Gitman, L. \& Joehnk, M. (2009). Fundamentos de inversiones. Pearson Educación.

Graham, B. \& Dodd, D. (2009). Security Analysis. Principles and Technique. McGraw-Hill. 
Hosseini, J. (1997). Cognitive dissonance as a means of exmplaining economics of irrationality and uncertainty. Journal of SocioEconomics, 26(2), pp.181-189.

La República. (2018). Odebrecht sigue afectando el precio de las acciones del mayor grupo bancario del país. Larepublica.co. https://www.larepublica.co/economia/odebrecht-sigue-afectandoa-mayor-grupo-bancario-de-colombia-2763211

Malkiel, B. (2003). The efficient market hypothesis and its critics. Journal of Economic Perspectives, 17(1), pp.59-82.

Mascareñas, J. (2007). Principios de finanzas. Universidad Complutense de Madrid.

Miles, M. \& Huberman, M. (1984). Qualitative data analysis. A source book of new methods, Beverly Hills, Sage. "Data management and analysis methods". En Denzin y Lincoln (eds.), Handbook of cualitative research. Londres: Sage Publication.

Murphy, J. (2000). Análisis técnico de los mercados financieros. New York Institute of Finance.

Nieto, B. \& Rodríguez, R. (2005). Modelos de valoración de activos condicionales: un panorama comparativo. Investigaciones Económicas, 29(1), 33-71.

Kahneman, D. \& Tversky, A. (1981). The framing of decisions and the psychology of choice. Science, 211(30), 453-458

Ofek, E. \& Richardson, M. (2003). Dotcom Mania. The rise and fall of internet stock prices. Journal of Finance, 58(3), 1113-1137.

Quiroga, E. (2017). Eficiencia en los mercados financieros y predicción de precios de los activos. Ciencias Administrativas, 10, 47-53.

Radim, G. (2012). The use of indicators in modified historical model to estimate the intrinsic value of a stock. Journal of Competitiveness, $4(2), 97-110$. 
Sharpe, A. (2003). Fundamentos de inversiones. Teoría y práctica. Prentice Hall.

Shiller, R. (2008). The suprime solution. Princenton University Press.

Shiller, R. (2013). Exuberancia irracional. Deusto.

Simon, H. (1990). Bounded Rationality. Palgrave Macmillan.

Thaler, R. (1980). Toward a positive theory of consumer choice. Journal of Economic Behavior and Organization, 1, 39-60.

Uribe, J. \& Ulloa, I. (2011). Revisando la hipótesis de los mercados eficientes: nuevos datos, nuevas crisis y nuevas estimaciones. Cuadernos de Economía, 30(55), 127-154.

Vilariño, A. (2013). Los mercados financieros son eficientes... para algunos. Revista de Economía Crítica, 16, 4-17

Zuluaga, M. \& Velázquez, J. (2007). Selección de indicadores técnicos para la negociación en el mercado cambiario colombiano I. Comportamientos individuales, 74(152), 9-20. 